\title{
REPORTE DE CASO
}

\section{Hepatectomía derecha para lesión del conducto biliar posterior a colecistectomía}

\author{
Right hepatectomy for post-cholecystectomy bile duct injury
}

\author{
Arellano, Nelson Dario1; $\stackrel{\oplus}{\text { Páez, Larissa Inés² }}$
}

'Universidad Nacional de Asunción, Facultad de Ciencias Médicas, Hospital de Clínicas, Unidad de Trasplante Hepático. San Lorenzo, Paraguay.

¿Universidad Nacional de Asunción, Facultad de Ciencias Médicas, Hospital de Clínicas, Segunda Cátedra de Clínica Quirúrgica. San Lorenzo, Paraguay.

Como referenciar éste artículo | How to reference this article:

Arellano ND, Páez LI. Hepatectomía derecha para lesión del conducto biliar posterior a colecistectomía. An. Fac. Cienc. Méd. (Asunción), 2021; 54(3): 161-166

\section{RESUMEN}

Mujer de 54 años sometida a colecistectomía laparoscópica programada por colecistolitiasis sintomática, el procedimiento fue convertido a abordaje abierto por presencia de bilirragia perioperatoria no localizada, tratada con sutura primaria del lecho vesicular bajo sospecha de un conducto aberrante de Luschka.

Al vigésimo día postoperatorio se diagnostica una fístula biliar tras la aparición de bilirragia a través de la herida quirúrgica. Una colangiografía transhepática percutánea mostró una pérdida completa de continuidad con fuga a ese nivel, confirmando la lesión del conducto hepático derecho, con un extremo cortado retraído del conducto hepático. Debido a la compleja lesión de la vía biliar proximal, a los 3 meses de la primera cirugía se realiza una hepatectomía derecha. Aunque la hepatectomía no es un procedimiento estándar para pacientes con lesiones quirúrgicas de la via biliar, debe considerarse como parte del arsenal quirúrgico para la reparación de un grupo seleccionado de pacientes en lesiones postcolecistectomía.

Palabras Clave: Hepatectomía, Lesión de las vías biliares, Colecistectomía.

Autor correspondiente: Dra. Larissa Inés Páez López. Médico Residente, Segunda Cátedra de Clínica Quirúrgica, Universidad Nacional de Asunción, Facultad de Ciencias Médicas, Hospital de Clínicas. San Lorenzo, Paraguay. E-mail: laripaez17415@gmail.com

Fecha de recepción el 27 de Febrero del 2021; aceptado el 11 de Mayo del 2021. 


\section{ABSTRACT}

A 54-year-old female underwent a planned laparoscopic cholecystectomy due to a symptomatic cholecystolithiasis, the procedure was converted to an open approach due to the presence of a not located perioperative bilirhagia, treated with a primary suture of the gallbladder bed under the suspicion of an aberrant duct of Luschka.

On the 20th postoperative day, the patient is diagnosed with a biliary fistula after the appearance of bilirhagia through the surgical wound. A percutaneous transhepatic cholangiography showed a complete loss of continuity with leakage at that level, confirming the right hepatic duct injury, with a retracted cut end from the hepatic duct. Due to the complex proximal bile duct injury, 3 months after the first surgery, a right hepatectomy is performed. Although an hepatectomy is not a standard procedure for patients with IBDI, it should be considered as a part of the surgical armamentarium for the repair of a selected group of patients in postcholecystectomy injuries.

Keywords: Hepatectomy, Bile duct Injury, Cholecystectomy.

\section{INTRODUCCION}

La colecistectomía es uno de los procedimientos quirúrgicos más comunes realizados por un médico de cirugíageneral. Debidoala aceptación mundial de los enfoques laparoscópicos en el tratamiento de los cálculos de la vesícula biliar, la incidencia de lesiones intraoperatorias ha aumentado (1). A pesar de las mejoras en las habilidades quirúrgicas y la experiencia en colecistectomía laparoscópica (CL), la tasa de lesión de la vía biliar (LVB) es aún mayor que en la cirugía abierta, permaneciendo para diferentes series entre 0.16 y $1.4 \%$ y 0.07 y $0.3 \%$ para ambos procedimientos, respectivamente (2).

Las lesiones quirúrgicas de las vías biliares (LQVB) siguen siendo un desafío para los cirujanos. Las complicaciones asociadas pueden manifestarse como eventos tempranos o tardíos. Las complicaciones tempranas comunes son la fuga de bilis, ictericia causada por obstrucción e isquemia hepática o de los conductos biliares después de una lesión vascular. Además de ser una consecuencia de un intento de reparación primaria inadecuado, las complicaciones tardías comprenden estenosis biliares y cálculos intraductales después de estasis biliar, colangitis recurrente, fibrosis o cirrosis biliar y atrofia lobar (3).
Se recomiendan técnicas endoscópicas como tratamiento inicial de LQVB. Cuando el tratamiento endoscópico no es efectivo, se considera la cirugía. En la mayoría de los pacientes en LQVB se realizan diferentes reconstrucciones biliares quirúrgicas. La hepaticoyeyunostomía de Roux-Y es la reconstrucción biliar más común para la LQVB. En algunos pacientes con LQVB compleja, se requiere hepatectomía (1).

Un estudio que analiza los factores de riesgo que llevaron a la hepatectomía en pacientes con LQVB después de colecistectomía laparoscópica (CL) concluye que la LQVB proximal (que involucra confluencia hepática) y la LQVB asociada a lesiones vasculares fueron los dos factores de riesgo independientes de hepatectomía y distinguieron dos grupos principales de pacientes que requieren resección hepática en LQVB: aquellos con una necrosis hepática inducida por lesión que requiere una intervención temprana y aquellos en quienes la resección hepática está indicada para el tratamiento de la atrofia hepática después de una colangitis prolongada [5]. Presentamos un caso que se resolvió en una hepatectomía derecha posterior a una colecistectomía laparoscópica. 


\section{Presentación del Caso}

Una mujer de 54 años se sometió a una colecistectomía laparoscópica programada, debido a una colecistolitiasis sintomática. El procedimiento se convirtió a colecistectomía abierta por la presencia de bilirragia perioperatoria no localizada, tratada con sutura primaria del lecho vesicular bajo sospecha de conducto aberrante de Luschka, así como la colocación de un drenaje transcístico con salida de una media de $400 \mathrm{cc}$ de bilis cada 24 horas.

El paciente es dado de alta al $9^{\circ}$ día postoperatorio, con ecografía abdominal y analítica de función hepática sin hallazgos relevantes, así como una leve leucocitosis de 13.000 células / $\mu \mathrm{L}$. Debido a la aparente secreción biliar en el lecho quirúrgico, se realiza una colangiopancreatografía retrógrada endoscópica (CPRE) al $11^{\circ}$ día postoperatorio, bajo sospecha de fuga del conducto cístico, y se trata con un stent biliar. Al día siguiente, la ecografía mostró un bilioma y una colección subhepática.

Con signos de sepsis, en el día 14 del postoperatorio, la paciente es sometida a una laparotomía exploradora y toilette, luego de lo cual es trasladada a la unidad de cuidados intensivos donde también se le diagnostica una infección del sitio quirúrgico.

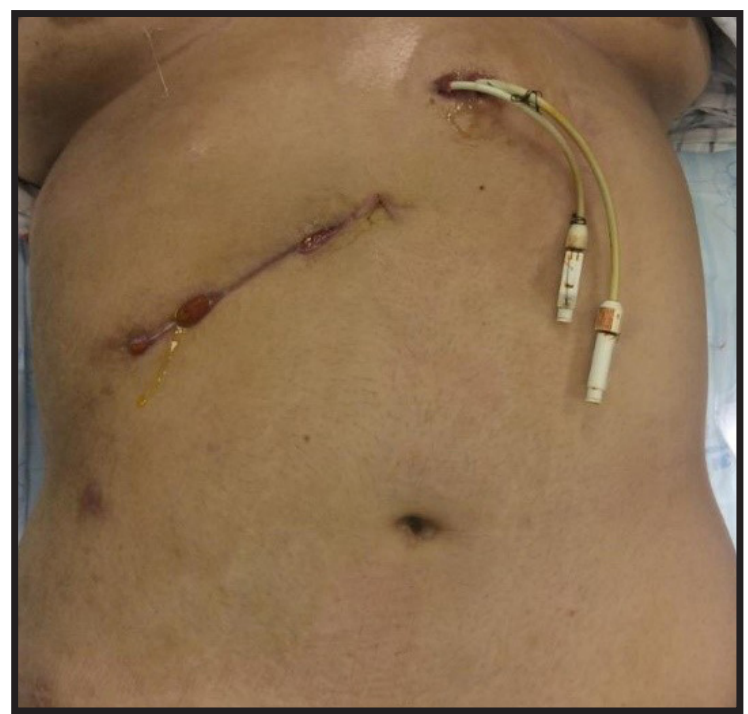

Figura 1. Incisión subcostal de Kocher, con aparente fuga de bilis. Drenajes percutáneos en el sitio.
Al vigésimo día postoperatorio la paciente presentó bilirragia a través de la herida quirúrgica subcostal, así como alrededor del drenaje biliar. La Resonancia Magnetica mostró un bilioma y una lesión biliar que afectaba al conducto hepático derecho, con pérdida de continuidad (Stewart IV, sin lesión vascular incluida). Al día siguiente, se colocó un drenaje percutáneo (pigtail de 10-12F) como tratamiento del bilioma, con una salida de aprox. $550 \mathrm{cc}$ de bilis al día.

Tras el traslado a un centro de referencia, una colangiografía transhepática percutánea mostró un corte completo con fuga a ese nivel, confirmando la lesión del conducto hepático derecho, con un extremo cortado retraído del conducto hepático (Figura 2).

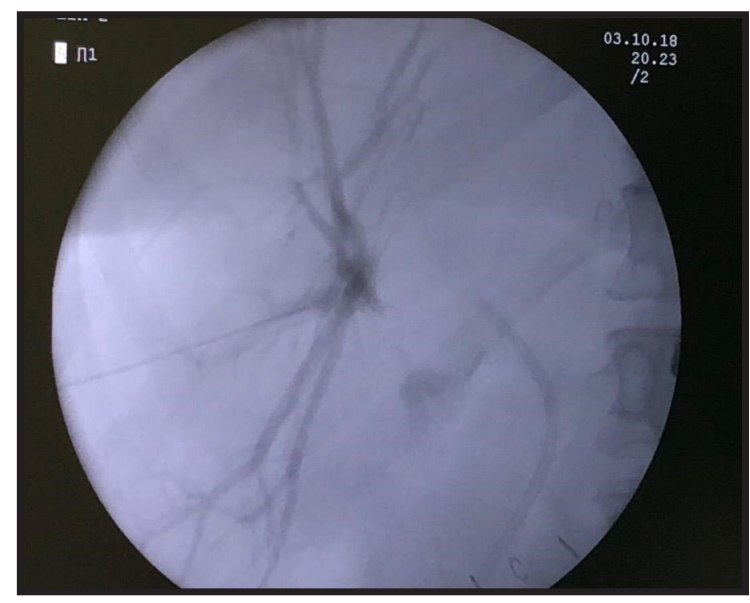

Figura 2. Colangiofrafia tranhepatica.

Ante la imposibilidad de realizar una cirugía de derivación biliodigestiva por retracción y reacción inflamatoria severa, se decide realizar una hepatectomía derecha a los 3 meses de la primera cirugía (Figura 3 - Figura 4). El paciente es dado de alta a la semana del postoperatorio, sin complicaciones. 


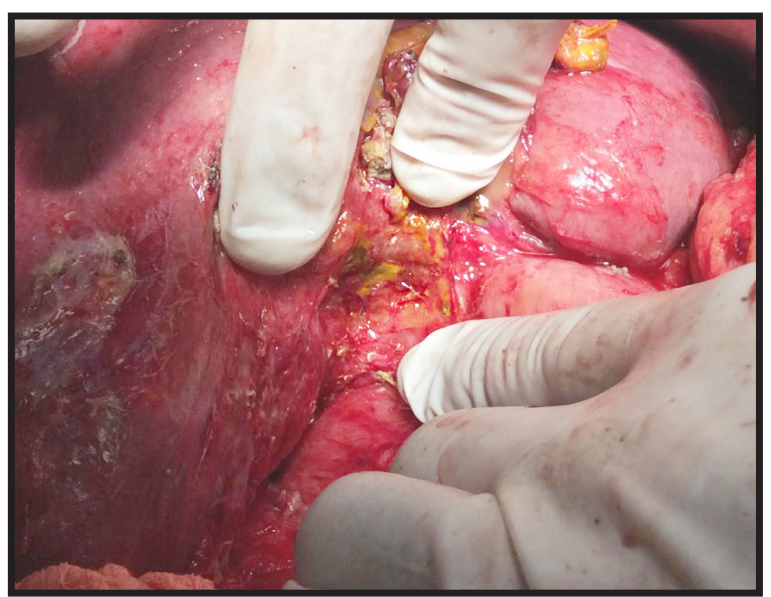

Figura 3. (izquierda) Retracción e inflamación severa del conducto hepático común.

\section{DISCUSION}

Este caso clínico se presenta en un intento de hacer referencia a la posibilidad de una hepatectomia como ultima herramienta a ser tenida en cuenta como parte del arsenal del manejo de una LQVB constatada o no desde una primera instancia por parte de un cirujano general y los factores de riesgo para llegar a la misma. Hace hincapié además en el difícil manejo y diagnóstico preciso de las mismas, como en este caso. Según De Santibáñes (4), solo entre el $15 \%$ y el $30 \%$ de las lesiones biliares se diagnostican durante el procedimiento quirúrgico y el cirujano debe considerar cuidadosamente su experiencia y capacidad para reparar cualquier lesión que sea inmediatamente identificable durante el procedimiento laparoscópico o mediante la conversión a laparotomía abierta; las lesiones deben ser reparadas preferiblemente por un cirujano experimentado en cirugía hepatobiliar.

Truant (6), mostró que las lesiones biliares proximales con interrupción de la confluencia ductal hepática (Strasberg tipos E4 O E5) y / o lesiones arteriales concurrentes son factores independientes para la hepatectomía en el tratamiento de la lesión de via biliar postcolecistectomía. Entre 31 estudios, el 92,6\% de 99 pacientes sometidos a hepatectomía tenían una lesión biliar compleja subyacente. Los pacientes con lesiones combinadas

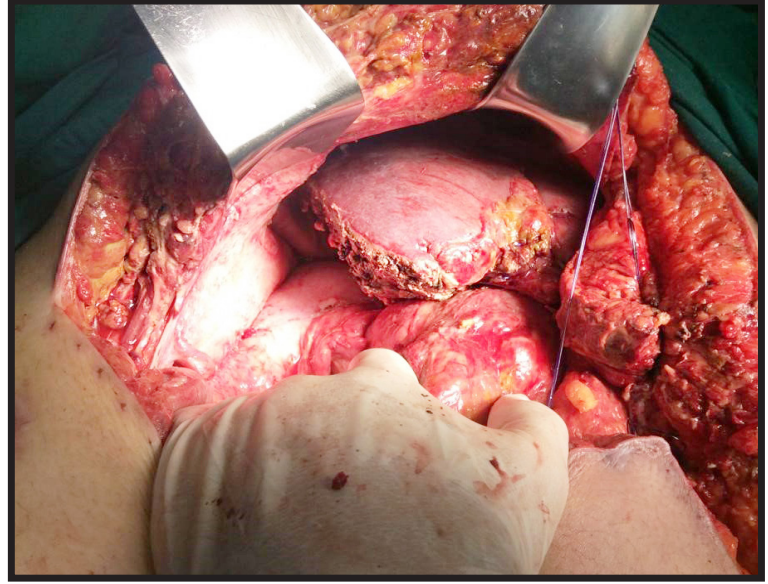

Figura 4. (derecha) Hepatectomia derecha.

arteriales y de Strasberg E4 o E5 tenían un riesgo particular de hepatectomía, con una razón de probabilidades de 43,3 (IC del 95\%: 8,0 a 234,2), en comparación con los pacientes sin ninguna de estas lesiones complejas.

Las indicaciones para una hepatectomía urgente pueden ser cuestionables, excepto en casos de lesiones arteriales y de la vena porta concomitantes. Cuando la lesión arterial es el único criterio de gravedad, su función hipotética como factor de hepatectomía urgente debe ser objeto de precaución (1).

La necesidad de hepatectomía se asoció, con una mayor tasa de reconstrucción biliar previa, cada reparación fallida se asocia de hecho con alguna pérdida de longitud de la vía biliar y requiere una disección más sustancial en el pedículo, con daño posterior a la vascularización de la vía biliar (6).

Stewart (2) estudió el impacto de la propagación de la lesión en operaciones sucesivas (es decir, el aumento de la extensión proximal de la LVB entre la lesión inicial y la posterior reconstrucción exitosa) sobre la incidencia de lesión arterial hepática. Para las lesiones ubicadas en o por encima de la bifurcación del conducto hepático común, el $49 \%$ de las que no se propagaron tenían lesión de la arteria hepática derecha 
en comparación con el $71 \%$ de las que tenían propagación de la lesión $(P<0,05)$.

Li (3), distinguió dos grupos de pacientes con IBDI que requieren hepatectomía: aquellos con una necrosis hepática inducida por lesión que requiere una intervención temprana y aquellos en quienes la resección hepática está indicada para el tratamiento de la atrofia hepática después de una colangitis a largo plazo. Los autores observaron una alta morbilidad postoperatoria (60\%) y mortalidad (10\%), y resultados satisfactorios a largo plazo (con una mediana de seguimiento de 34 meses) con ningún síntoma o solo transitorios en el $67 \%$ de los pacientes.

Con base en los estudios de análisis en la base de datos PubMed, los autores presentaron las siguientes indicaciones para la hepatectomía: sepsis biliar recurrente, estenosis biliares causadas por colangitis continua, abscesos intrahepáticos, novisualizacióny/oinadecuación del muñón proximal del conducto o conductos biliares lesionados para anastomosis, lesiones intrahepáticas de un conducto hepático derecho aberrante, estenosis anastomóticas y litiasis intrahepática, atrofia lobar hepática derecha, cirrosis biliar secundaria y tumor de Klatskin primario no diagnosticado (4).

El diagnóstico precoz y adecuado de la LQVB es crucial para los cirujanos y gastroenterólogos, porque la LQVB no reconocida conduce a complicaciones graves como cirrosis biliar, insuficiencia hepática e incluso la muerte (3). Cuando el tratamiento endoscópico no es efectivo, se considera la cirugía.

La incidencia de lesiones biliovasculares complejas ha aumentado desde la introducción de la CL (4). Según el momento de la intervención quirúrgica, dos grupos de indicaciones (precoz y tardía) puede ser distinguido. En el postoperatorio temprano después de la colecistectomía, la hepatectomía es necesaria en pacientes con necrosis o abscesos hepáticos y fuga de bilis, para controlar la peritonitis y la sepsis. En los pacientes restantes, se requiere hepatectomía en casos de colangitis recurrente, que no responden al tratamiento estándar, y atrofia lobar sintomática.

Aunque la hepatectomia no es un procedimiento estándar para los pacientes con LQVB, debe considerarse como parte del arsenal quirúrgico para la reparación de un grupo seleccionado de pacientes en lesiones poscolecistectomía. Debido a su alta complejidad, estos pacientes deben ser manejados en centros de alta referencia (6).

\section{Conflicto de intereses y financiación:}

Los autores declaran no tener conflictos de interés, no estar involucrados como investigadores en ensayos clínicos en curso sobre el tema, y no haber recibido donaciones o beneficios por parte de los grupos interesados en las recomendaciones.

\section{Declaración de la contribución de autores y colaboradores:}

Nelson Darío Arellano Cabral: Cirujano a cargo del caso y tutor en la redacción del caso clínico

Larissa Inés Páez López: Residente a cargo, recolección de datos y redacción del caso clínico.

\section{REFERENCIAS BIBLIOGRÁFICAS}

1. 1. Deziel DJ, Millikan KW, Economou SG et al (1993) Complications of laparoscopic cholecystectomy: a national survey of 4,292 hospitals and an analysis of 77,604 cases. Am J Surg 165:9-14

2. 2. Archer SB, Brown DW, Smith CD et al (2001) Bile duct injury during laparoscopic cholecystectomy. Results of a National Survey. Ann Surg 234:549-559

3. 3. Li J, Frilling A, Nadalin S, Broelsch CE, Malago M. Timing and risk factors of hepatectomy in the management of complications following laparoscopic cholecystectomy. J Gastrointest Surg 2012; 16: 815-820 [PMID: 22068969 DOI:10.1007/s11605-011-1769-2].

4. 4. Jabłońska B. Hepatectomy for bile duct injuries: When is it necessary? World J Gastroenterol 2013; 19(38): 63486352 Available from: URL: http://www.wjgnet.com/10079327/full/ v19/i38/6348.htm DOI: http://dx.doi.org/10.3748/ wjg.v19. i38.6348.

5. 5. De Santibáñes, E., Ardiles, V., \& Pekolj, J. (2008). Complex bile duct injuries: management. HPB: The Official Journal of the International Hepato Pancreato Biliary Association, 10(1), 4-12. 
6. 6. Truant, S,Boleslawsk, E. Géraldine. Hepatic resection for post-cholecystectomy bile duct injuries:a literature review. Department of Digestive Surgery and Transplantation, University. Hospitals, Lille, France. HPB 2010, 12, 334-341

7. 7. Kayaalp C, Nessar G, Kaman S, Akoglu M. (2001) Right liver necrosis: complication of laparoscopic cholecystectomy. Hepatogastroenterology 48:1727-1729.

8. 8. Stewart L, Robinson TN, Lee CM, Liu K, Whang K, Way LW. (2004) Right hepatic artery injury associated with laparoscopic bile duct injury: incidence, mechanism, and consequences. J Gastrointest Surg 8:523-530; discussion 530-531.

9. 9. Negi SS, Sakhuja P, Malhotra V, Chaudhary A. Factors predicting advanced hepatic fibrosis in patients with postcholecystectomy bile duct strictures. Arch Surg 2004; 139: 299-303 [PMID: 15006888 DOI: 10.1001/archsurg.139.3.299]

10. 10. Addeo P, Oussoultzoglou E, Fuchshuber P, Rosso E, Nobilic, Souche R, Jaeck D, Bachellier P. Reoperative surgery after repair of postcholecystectomy bile duct injuries: is it worthwhile? World J Surg 2013; 37: 573-581 [PMID: 23188533 DOI:10.1007/s00268-012-1847-y]

11. 11. Pekolj, J. Major Liver Resection as Definitive Treatment in Post-cholecystectomy Common Bile Duct Injuries. World J Surg 2015:[ DOI 10.1007/s00268-014-2933-0]. 